Revista Universo Contábil, ISSN 1809-3337

Blumenau, v. 14, n. 3, p. 149-167, jul./set., 2018

doi:10.4270/ruc.2018324

Disponível em www.furb.br/universocontabil

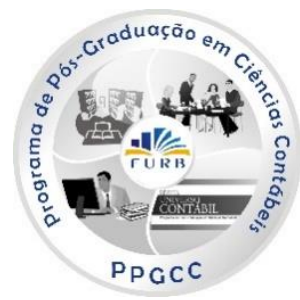

\title{
DISCLOSURE VOLUNTÁRIO VIA REDES SOCIAIS DAS EMPRESAS LISTADAS NO IBRX1001
}

\section{VOLUNTARY DISCLOSURE VIA SOCIAL NETWORKS OF COMPANIES LISTED IN IBRX100}

\section{DISCLOSURE VOLUNTARIO A TRAVÉS DE LAS REDES SOCIALES DE LAS EMPRESAS LISTADAS EN EL IBRX100}

\author{
Geisa Cassiana Paulino \\ Mestre em Ciências Contábeis pela Universidade Federal da Paraíba \\ Endereço: PPGCC, Campus I, Cidade Universitária \\ CEP: 58059900 - João Pessoa, PB - Brasil \\ E-mail: geisapcont@gmail.com \\ Telefone: (83) 32167582 \\ Genilda Soares da Silva \\ Mestre em Ciências Contábeis pela Universidade Federal da Paraíba \\ Endereço: PPGCC, Campus I, Cidade Universitária \\ CEP: 58059900 - João Pessoa, PB - Brasil \\ E-mail: genyss2@hotmail.com \\ Telefone: (83) 32167582
}

Luiz Felipe de Araújo Pontes Girão Doutor em Contabilidade pelo Programa Multi-Institucional de Pós-Graduação UnB/UFPB/UFRN Professor do PPG em Ciências Contábeis da Universidade Federal da Paraíba Endereço: PPGCC, Campus I, Cidade Universitária CEP: 58059900 - João Pessoa, PB - Brasil

E-mail: Ifapg@hotmail.com Telefone: (83) 32167582

\section{RESUMO}

O objetivo deste trabalho foi de analisar a relação entre as principais práticas de disclosure voluntário, através do Twitter e do Facebook, das empresas pertencentes ao Índice Brasil (IBrX 100), e a demanda dos investidores pela informação via internet. O disclosure voluntário foi analisado por meio de um índice de disclosure voluntário (IDV), que foi criado com a combinação do índice de disclosure ambiental (IDA), estruturado por Altoé, Panhoca e Espejo (2013), com o índice de sustentabilidade, desenvolvido por Callado (2010). Aplicou-se o modelo Tobit pela natureza de distribuição censurada da variável dependente (IDV). Como

1 Artigo recebido em 21/05/2018. Revisado por pares em 07/02/2019. Reformulado em 27/02/2019. Recomendado para publicação em 28/02/2019 por Moacir M. Rodrigues Jr. Publicado em 30/03/2019. Organização responsável pelo periódico: FURB. 
variáveis explicativas, utilizou-se o quantitativo de seguidores, de curtidas e da cobertura de analistas de mercado por empresa. Os resultados para a variável quantidade de seguidores proxy para a demanda dos usuários por informações corporativas via redes sociais - indicam que quanto mais seguidores, menor é o índice de diclosure voluntário de informações. Especificamente para este resultado, mesmo sem um teste explícito, pode-se afirmar que as empresas com maior número de seguidores são as com mais divulgações/publicações de outros tipos de informação (produtos, promoções) em suas redes sociais. No tocante ao número de curtidas efetuadas nas informações consideradas voluntárias, a relação positiva com o IDV sugere que o comportamento de visualização do usuário do Facebook e do Twitter, de informações ambientais, sociais e econômicas seria um fator determinante do nível de disclosure de informações voluntárias realizado pelas empresas.

Palavras-chave: Disclosure; Facebook; Redes sociais; Twitter.

\section{ABSTRACT}

This paper aimed to analyze the relations between the main practices of voluntary disclosure, through Twitter and Facebook of the companies belonging to the Brazil Index (IBrX 100), and the investors' demand for information via the Internet. We analyzed voluntary disclosure through a voluntary disclosure index, based the combination of the environmental disclosure index, structured by Altoé, Panhoca, and Espejo (2013), with the sustainability index developed by Callado (2010). It was applied the Tobit model by the nature of censored distribution of the dependent variable. We used as explanatory variables, the number of followers, tanners and market analysts' coverage per company. The results for the variable number of followers proxy for users' demand for corporate information via social networks - indicate that having more followers mean a lower number of voluntary disclosure of information. Specifically, for this result, even without an explicit test, it can be affirmed that the companies with the largest number of followers are those with the most divulgations/publications of other types of information (products, promotions) in their social networks. Regarding the number of likes in the information considered to be voluntary, the positive relationship with voluntary disclosure index suggests that the user's viewing behavior of Facebook and Twitter, environmental, social and economic information would be a determining factor in the level of disclosure of voluntary information carried out by the companies.

Keywords: Disclosure; Facebook; Social Medias; Twitter.

\section{RESUMEN}

El objetivo de este trabajo fue de analizar la relación entre las principales prácticas de disclosure voluntario, a través del Twitter y del Facebook, de las empresas pertenecientes al Índice Brasil (IBrX 100), y la demanda de los invertidores por la información a través de la internet. El disclosure voluntario fue analizado por medio de un índice de disclosure voluntario (IDV), que fue creado con la combinación del índice de disclosure ambiental (IDA), estructurado por Altoé, Panhoca y Espejo (2013), con el índice de sustentabilidad, desarrollado por Callado (2010). Se aplicó el modelo Tobit por la naturaleza de distribución censurada de la variable dependiente (IDV). Como variables explicativas, se utilizó el cuantitativo de seguidores, de curtidas y de la cobertura de analistas de mercado por empresa. Los resultados para la variable cuantidad de seguidores - proxy para la demanda de los usuarios por informaciones corporativas a través de las redes sociales - indican que cuanto más seguidores, menor es el índice de diclosure voluntario de informaciones. Específicamente para este resultado, mismo sin un teste explícito, se puede afirmar que las empresas con mayor número de seguidores son las con más divulgaciones/publicaciones de otros tipos de información (productos, rebajas) en sus redes sociales. A lo que toca el número de likes efectuados en las informaciones 
consideradas voluntarias, la relación positiva con el IDV sugiere que el comportamiento de visualización del usuario del Facebook y del Twitter, de informaciones ambientales, sociales y económicas sería un factor determinante del nivel de disclosure de informaciones voluntarias realizado por las empresas.

Palabras-clave: Disclosure; Facebook; Redes sociales; Twitter.

\section{INTRODUÇÃO}

O mercado de capitais pode ser entendido como um ambiente onde são realizadas transações entre os agentes deficitários (empresas) e os superavitários (investidores). Para estes últimos, a divulgação de informações financeiras pelas empresas, por meio dos canais tradicionais, é base para a tomada de decisão (ARRUDA; GIRÃO; LUCENA, 2015). A questão é que os meios tradicionais de divulgação de informações usados pelas firmas frequentemente só alcançam uma parte dos investidores, o que resultaria em informação assimétrica entre os agentes (BLANKESPOOR; MILLER; WHITE, 2013). A assimetria de informação é considerada um fenômeno resultante da separação da propriedade e da gestão (JENSEN; MECKLING, 1976), em que o nível de informação entre o gestor e o investidor é distinto, pois como este último está fora do ambiente da companhia, não tem acesso à informação na íntegra.

A presença da assimetria informacional entre vendedores e compradores acerca dos ativos transacionados é considerada um catalizador de problemas, como a seleção adversa e o risco moral (AKERLOF, 1970; ALBANEZ; VALLE, 2009; HEALY; PALEPU, 2001; PAULO, 2007), e pode afetar a precificação ou avaliação das empresas (GIRÃO; MARTINS; PAULO, 2013; MACAGNAN, 2009). A divulgação de informações seria uma das condições para reduzir a assimetria de informação e, por conseguinte, alocar eficientemente recursos (HEALY; PALEPU, 2001; MACAGNAN, 2009). Nesse contexto corporativo, a informação passa a ser desejada porque é útil. Então, espera-se que surja o interesse por sua regulamentação, o que, para Akerlof (1970), seria umas das formas de evitar o colapso do mercado.

Teoricamente, apontam-se diversos motivos que poderiam influenciar a gestão das empresas a evidenciar ou não informações de caráter voluntário. Verrecchia (2001) e Pae (2005) argumentam que um desses motivos é o interesse da gestão em evitar potenciais ameaças de litígios, em que um investidor poderia implementar uma ação legal contra a empresa por causa de divulgações inadequadas ou a falta delas, embora se saiba que mesmo as empresas que fazem um amplo disclosure ainda são consideradas pelo mercado como não totalmente transparentes sobre suas informações (PAE, 2005) e que a falta de divulgação voluntária por parte de uma firma é percebida pelos investidores como um prenúncio de más notícias (MENDES-DA-SILVA et al., 2009). Em contrapartida, quanto mais as empresas divulgam informações relevantes, confiáveis e adicionais às obrigatórias, mais bem vistas se tornam no mercado (FORTE et al., 2014; HUGHES; PAE, 2004). Assim, o disclosure voluntário pode ser encarado como algo dinâmico, em que as empresas desenvolvem estratégias em busca de um ponto ótimo de divulgação, e não, um ponto máximo, e escolhem o que divulgar, quando e como (PAE, 2005).

Nesse contexto de diclosure corporativo, adicione-se o desenvolvimento de tecnologias que contribuíram para o surgimento das mídias sociais, que podem ser usadas pelas firmas como um canal adicional de divulgação (BLANKESPOOR; MILLER; WHITE, 2013). Há, ainda, um uso recorrente desses canais pelas empresas para divulgar informações e fatos relevantes (ARRUDA; GIRÃO; LUCENA, 2015) corporativos de forma mais tempestiva, já que o custo de divulgação para a empresa seria mais baixo em comparação com o dos canais tradicionais e reduz o custo de aquisição de informação pelo investidor (BLANKESPOOR; MILLER; WHITE, 2013), que pode ser considerado um dos principais interessados no disclosure corporativo e, por essa razão, teria interesse em acompanhar as publicações das empresas via 
mídias sociais. Dessa forma, a internet passa a ser um importante canal por meio do qual os investidores expressam sua demanda por informações públicas (DRAKE; THORNOCK; ROULSTONE, 2012).

Considerando que o disclosure voluntário é importante para o mercado de capitais, já que reduz a assimetria informacional e, por conseguinte, diminui os problemas de seleção adversa e risco moral, devido ao surgimento das mídias sociais como um canal alternativo com menos custo de disclosure para as empresas, assim como menos exigência de esforço para os investidores obterem essas informações, este estudo teve o objetivo de analisar a relação entre as principais práticas do disclosure voluntário, através do Twitter e do Facebook, das empresas pertencentes ao Índice Brasil (IBrX 100) e a demanda dos investidores pela informação via internet.

A atenção que esta pesquisa deu ao tema do disclosure voluntário das empresas via web surgiu do interesse de analisar o tipo de informação publicado pelas empresas em suas redes sociais e se isso está relacionado à capacidade de ampla divulgação (medido pela quantidade de seguidores e de curtidas) que desses canais alternativos. Assim, esta pesquisa pretende contribuir com os estudos sobre os fatores determinantes da disseminação voluntária via internet, porque, no Brasil, essa questão é pouco explorada (MENDES-DA-SILVA et al., 2009).

A amostra inicial foi composta de todas as empresas que fazem parte do Índice Brasil (IBrX 100) da Brasil Bolsa Balcão (B3). Posteriormente, procurou-se saber quais as empresas que mantinham conta de Twitter e/ou de Facebook ativa no ano de 2016. Assim, foram analisadas 60 páginas de Facebook e 52 de Twitter em busca de informações consideradas voluntárias, de acordo com um índice de disclosure voluntário construído. O modelo econométrico usado foi a regressão Tobit, por causa da característica de censura da variável dependente.

$\mathrm{Na}$ análise dos resultados, esperava-se uma relação positiva entre o IDV e o número de seguidores e curtidas nas informações voluntárias divulgadas. Outra hipótese testada foi sobre a cobertura de analistas de mercado e o comportamento de disclosure voluntário realizado pelas empresas via redes sociais. Os achados indicaram que a quantidade de analistas, apesar de apresentar uma relação negativa com o nível de disclosure voluntário, não tem poder estatístico para influenciar o tipo de divulgação feito pelas empresas nas redes sociais analisadas.

\section{REFERENCIAL TEÓRICO}

\subsection{Disclosure voluntário}

A partir de um enfoque informacional, Mendes-da-Silva et al. (2009) afirmam que a falta de divulgação voluntária é vista pelos investidores como um prenúncio de más notícias. Por outro lado, quanto mais as empresas divulgam informações relevantes, mais bem vistas ficam no mercado (ARRUDA; GIRÃO; LUCENA, 2015). Nesse contexto, as empresas passaram a incorporar mais transparência na divulgação de informações de forma voluntária, como complemento ao fornecimento de informações obrigatórias, porém de forma estratégica, pois, conforme Bertomeu e Magee (2015), há evidências de que existe um ponto de equilíbrio entre o disclosure voluntário e o disclosure obrigatório traçado pelas empresas.

As evidências de informações voluntárias pelas Entidades são abordadas pela Teoria da Divulgação, em que alguns dos principais trabalhos são os seminais de Verrecchia (1983) e Dye (1985). Esses autores tratam das circunstâncias que cercam a decisão do administrador em divulgar ou não uma informação para o público, visto que os administradores detêm informações privadas acerca da empresa, cuja divulgação não é obrigatória, por isso analisam as circunstâncias favoráveis a essa divulgação. 
Autores como Botosan (1997), Hail (2002), Chen, Chen, Wei (2003), Botosan, Plumlee (2005) e Alencar (2007) apontam benefícios da divulgação de informações e predizem, entre outros pontos, que o maior nível de disclosure reduz o custo do capital próprio. Já Welker (1995), Healy, Hutton \& Palepu (1999), Leuz, Verrecchia (2000) e Zhou (2004) apontam, entre outros aspectos, que um nível maior de disclosure reduz, especificamente, o custo do capital de terceiros (BUSHEE; NOE, 2000). Os achados desses trabalhos justificariam um disclosure maior por parte das empresas.

Ressalte-se, no entanto, que um dos motivos apontados pela literatura para as empresas não adotarem a política do disclosure completo é a questão dos custos. Verrecchia (2001) argumenta que a falta de custos de divulgação pressupõe uma divulgação completa da informação, enquanto, para Dye (2001), à medida que os custos de divulgação aumentam, o disclosure voluntário tende a diminuir. Porém as empresas, atualmente, têm possibilidade de traçar estratégias e divulgar suas informações, por meio das redes sociais, referentes aos aspectos econômicos, sociais e ambientais, de forma voluntária, com o fim de obter vantagem competitiva por incorrer em menores custos de divulgação. Segundo Zhang (2015), as empresas já utilizam gradativamente as redes sociais como forma de se comunicar com seus investidores, cujo conteúdo divulgado pode, inclusive, afetar a decisão desses investidores.

Blankespoor, Miller e White (2013) explicam que o uso das redes sociais vem crescendo como um canal alternativo para divulgar informações e fatos relevantes de forma voluntária, tempestiva e com baixo custo. Drake et al. (2012) enfatizam que os investidores expressam suas demandas por informações através da internet e reforçam a importância do disclosure completo pelas empresas.

Das organizações que se comprometem com a sustentabilidade espera-se um nível maior de divulgação, pois, como referem Gray e Bebbington (2000), há benefícios como favorecimento de uma imagem corporativa, legitimação de suas atividades, antecipação de ações regulatórias, impacto positivo no preço das ações bem como vantagens competitivas. Essa disseminação de informações, segundo Bushman, Chen, Engel e Smith (2004), melhora a transparência da empresa.

Congruente com esse ponto, o tamanho da empresa, sob o ponto de vista de Ahmed e Courtis (1999) e de Forte et al. (2014), é considerado uma variável relevante, que explica o disclosure. Segundo Blankespoor et al. (2013), o disclosure via redes sociais, como, por exemplo, Twitter, faz com que as empresas com mais visibilidade (maiores) sejam mais propícias a divulgar menos informações voluntárias em relação às demais empresas, porque já são cobertas pela mídia tradicional.

Em relação ao endividamento, e baseando-se na Teoria da Agência como instrumento de redução dos custos de agência da dívida, os gestores mais endividados procuram evidenciar mais informações para satisfazer aos credores e remover as suspeitas sobre a transferência de riqueza para o acionista (JENSEN; MECKLING, 1976; MURCIA; SANTOS, 2009). Nesse contexto, é importante mencionar também que, embora as pesquisas apontem um crescimento no uso das redes sociais, algumas empresas ainda são cautelosas ao utilizar essa ferramenta como canal de divulgação de suas informações, pois, para Zhang (2015), algumas empresas estão preocupadas com as incertezas das redes sociais e se mantêm relutantes sobre a divulgação de informações por meio de tais canais.

\subsection{Mídias sociais e disclosure voluntário}

$\mathrm{Na}$ última década, a internet tornou-se uma fonte cada vez mais importante de recolhimento de informações sobre as empresas (RUBIN; RUBIN, 2010). As novas tecnologias, principalmente as plataformas de mídias sociais, segundo Zhang (2015), possibilitaram o acesso de pessoas à informação de mercado e a novas notícias em segundos. Características como menores custos de divulgação de informações sobre suas transações, o 
valor de suas cotações, marketing de vendas, entre outras (ARRUDA et al., 2015), podem ser consideradas como incentivo para que as empresas usem a web como canal de divulgação. Outro fator a ser considerado é a crescente busca dos investidores por mais informações sobre as empresas (RUBIN; RUBIN, 2010) que encontram nas redes sociais - quando a informação é implantada - um amplo volume de divulgações à disposição (ARRUDA et al., 2015).

Autores como Zhang (2015) investigaram a adoção de novos meios de comunicação pelas companhias para divulgar informações para os consumidores e os investidores. Seus achados sugerem que o engajamento de divulgação de informações atráves de novos meios de comunicação aumenta a influência e o alcance da empresa. Assim, o uso das mídias sociais por parte das empresas como mais um canal de comunicação com os seus stakeholders (SILVA, M. D. O. P.; LUCENA, 2015) despertou o interesse acadêmico para o efeito dessas práticas na precificação de ações (ARRUDA; GIRÃO; LUCENA, 2015; SILVA, M. D. O. P.; LUCENA, 2015) e na redução da assimetria da informação (ARRUDA; GIRÃO; LUCENA, 2015; BLANKESPOOR; MILLER; WHITE, 2013), na associação entre as características das empresas e na disseminação voluntária de informações de interesse dos investidores (MENDES-DA-SILVA et al., 2009; SILVA, W. M. DA; MARTELANC, 2006).

Na perspectiva do investidor, Drake et al. (2012) consideram a internet um canal em que as informações públicas são disseminadas para investidores, e o volume de pesquisas no site Google seria uma proxy para expressar essa demanda pela informação. Um dos resultados de seu estudo em relação a anúncios de lucros sugere que os investidores começam a buscar e a processar informações através da internet nos dias que antecedem o anúncio de lucro, durante o anúncio, e duas semanas depois.

Silva e Lucena (2015), ao investigar a relação entre a divulgação de informações socioambientais nas redes sociais e a precificação das ações das empresas integrantes do IBrX 100 da Brasil Bolsa Balcão (B3), encontraram uma relação positiva entre as informações divulgadas na rede social Facebook e a precificação das ações. Abdelsalam, Bryant e Street (2007) examinaram a abrangência e os determinantes do Relatório Corporativo de Internet (CIR), dentro do novo ambiente regulatório da Bolsa de Londres, e concluíram que as empresas precisam melhorar a CIR, especialmente no que diz respeito à credibilidade das informações fornecidas nos sites corporativos e à usabilidade dos sites.

Pelos achados dos estudos supracitados, vê-se que a Internet passou a ser considerada como um canal de divulgação corporativa por meio do qual as empresas podem dar informações amplas e instantâneas, e isso pode justificar o despertar do interesse acadêmico a respeito dos determinantes e dos impactos dessa nova forma de disclosure direto.

\section{METODOLOGIA}

\subsection{Amostra e coleta dos dados}

Este estudo investigou o disclosure voluntário, de acordo com o índice de disclosure voluntário (IDV) descrito na Tabela 2, realizado no Twitter e no Facebook, durante todo o ano de 2016, pelas empresas que compõem o IBrX 100 da B3. O uso dessas duas redes sociais se justifica porque, de acordo com os dados do Alexa (2013), elas estão entre as redes de relacionamento mais utilizadas pelas empresas no Brasil e no mundo.

As informações foram coletadas de forma manual e diretamente na conta do Twitter e do Facebook de cada empresa, entre os dias 15/01/2017 e 10/02/2017. Para isso, procedeu-se a uma análise de conteúdo e sistematizou-se a natureza das mensagens. Para Beretta e Bozzolan (2007), a análise de conteúdo é o método mais utilizado nos estudos sobre disclosure, pois dá credibilidade e inferências para a análise de acordo com determinado contexto. A Tabela 1 traz a composição final da amostra. 
Tabela 1 - Composição final da amostra

\begin{tabular}{lc}
\hline Descrição da amostra - Facebook & Quantidade \\
\hline Total de empresas do Ibrx 100 & 100 \\
Exclusão de empresas com mais de um tipo de ação cotada & $(6)$ \\
Exclusão de empresas sem Facebook & $(19)$ \\
Exclusão de empresas sem dado financeiro & $(11)$ \\
Exclusão de empresas sem informações de analistas & $(4)$ \\
& \\
Total da amostra & 60 \\
\hline Descrição da amostra - Twitter & Quantidade \\
\hline Total de empresas do Ibrx 100 & 100 \\
Exclusão de empresas com mais de um tipo de ação cotada & $(6)$ \\
Exclusão de empresas sem Twitter & $(32)$ \\
Exclusão de empresas sem dado financeiro & $(6)$ \\
Exclusão de empresas sem informações de analistas & $(4)$ \\
Total da amostra & 52 \\
\hline
\end{tabular}

Fonte: Dados da pesquisa (2017)

\subsection{Hipótese do modelo}

A relação construída foi baseada nos achados de Blankespoor et al. (2013) em relação à disseminação de informações via Twitter, e na pesquisa de Drake et al. (2012), no tocante à demanda de informações pelos investidores via internet.

Para as variáveis independentes, 'quantidade de seguidores' e 'curtidas', coletadas diretamente no Twitter e no Facebook das empresas, espera-se uma relação positiva com o IDV, pois Drake et al. (2012) encontraram evidências de que a internet é um canal em que os investidores expressam sua demanda por informações sobre as empresas. Então, espera-se que empresas com amplo disclosure tenham mais seguidores, que estariam interessados em se manter atualizados utilizando um canal barato, quando comparado com os demais, e rápido, de maneira que possam se beneficiar desse disclosure direto e tempestivo das empresas via Twitter e Facebook. Logo, a hipótese testada foi:

H1: Empresas com maior demanda de usuários no Twitter e no Facebook evidenciam um maior disclosure voluntário.

Essa demanda foi representada pela quantidade de seguidores e de curtidas de divulgações voluntárias, porque, quanto mais seguidores a empresa tiver nessas redes sociais, maior será o alcance de suas publicações. Em relação às curtidas, representam o número de seguidores que estariam lendo a informação divulgada. Adicionalmente, foi construída uma segunda hipótese de pesquisa. Para Blankespoor et al. (2013), a direta e ampla disseminação de informações via redes sociais (mais precisamente, o Twitter) seria mais benéfica para as empresas que são pouco visadas do que para as que são largamente acompanhadas pelos meios tradicionais de divulgação, como os analistas de mercado. Assim, espera-se que as empresas que tiverem menos analistas acompanhando suas divulgações sejam mais propícias a usar o Twitter e/ou o Facebook como meios de divulgar suas informações.

H2: Empresas mais visadas pelos analistas de mercado evidenciam menor disclosure voluntário. 


\subsection{Definição das variáveis do modelo empregado}

Com o objetivo de relacionar o disclosure voluntário direto com a demanda dos investidores por informações, pôs-se em questão o fato de a variável disclosure não ser observável, razão por que se optou por usar o índice de disclosure voluntário que advém da adaptação de dois índices já descritos na literatura pertinente, o índice de disclosure ambiental (IDA), estruturado por Altoé, Panhoca e Espejo (2015), e o índice de sustentabilidade (IS), criado por Callado (2010). Justifica-se esse procedimento pelo fato de que o IDA trabalha a questão ambiental e o IS trabalha, para além da questão ambiental, as dimensões sociais e econômicas. Assim, optou-se por criar um índice com a adaptação desses dois: o índice de disclosure voluntário (IDV), estruturado conforme a tabela 2.

Tabela 2 - Índice de disclosure voluntário (IDV)

\begin{tabular}{|c|c|c|c|}
\hline \multirow{2}{*}{\multicolumn{2}{|c|}{ Categoria ambiental - A empresa divulgou informações sobre }} & \multirow{2}{*}{ Sim } & \multirow[t]{2}{*}{ Não } \\
\hline & & & \\
\hline $\begin{array}{l}01 . \\
\text { atm }\end{array}$ & $\begin{array}{l}\text { Há informações sobre impactos ambientais dos produtos e processos (po } \\
\text { rica, das águas, sonora, visual)? }\end{array}$ & & \\
\hline 02. & Há informações sobre resíduos e desperdícios? & & \\
\hline 03. & Informou sobre uso eficiente/reutilização da água? & & \\
\hline 04. & As metas e os objetivos ambientais estão estabelecidos? & & \\
\hline 05 . & Há declarações sobre as políticas empresariais ambientais? & & \\
\hline 06. & A empresa informou sobre programa de gestão ambiental (longo prazo)? & & \\
\hline 07. & Informou sobre auditoria ambiental? & & \\
\hline 08. & Há informações sobre práticas contábeis de itens ambientais? & & \\
\hline 09. & Divulgou sobre reserva para proteção ambiental? & & \\
\hline 10. & Há informações sobre custos e/ou despesas ambientais? & & \\
\hline \multicolumn{4}{|c|}{ Categoria social - A empresa divulgou informações sobre } \\
\hline 11. & Geração de trabalho e renda? & & \\
\hline 12. & Auxílio em educação e treinamento? & & \\
\hline 13. & Padrão de Segurança do Trabalho? & & \\
\hline 14. & Ética organizacional? & & \\
\hline 15. & Integração social? & & \\
\hline 16. & Empregabilidade e gerenciamento de fim de carreira? & & \\
\hline 17. & Conduta de padrão internacional? & & \\
\hline 18. & Capacitação e desenvolvimento de funcionário? & & \\
\hline 19. & Acidentes fatais? & & \\
\hline 20. & Contratos legais? & & \\
\hline 21. & Stress de trabalho? & & \\
\hline 22. & Segurança do produto? & & \\
\hline \multicolumn{4}{|c|}{ Categoria econômica - A empresa divulgou informações sobre } \\
\hline 23. & Investimentos éticos? & & \\
\hline 24. & Gastos em saúde e segurança? & & \\
\hline 25. & Investimento em tecnologia limpa? & & \\
\hline 26. & Nível de endividamento? & & \\
\hline 27. & Lucratividade? & & \\
\hline 28. & Participação de mercado? & & \\
\hline 29. & Passivo ambiental? & & \\
\hline 30. & Avaliação de resultado da organização? & & \\
\hline 31. & Volume de vendas? & & \\
\hline 32. & Gastos com benefícios? & & \\
\hline 33. & Selos de qualidade? & & \\
\hline
\end{tabular}

Fonte: Adaptado de Callado (2010) e Altoé, Panhoca e Espejo (2013) 
Em cada categoria, a nota obtida pela empresa foi calculada dividindo-se a soma dos pontos obtidos pela pontuação total possível. O mesmo procedimento foi adotado para o cálculo total do IDV.

Como técnica estatística, adotou-se o modelo de regressão Tobit, pois, conforme Gujarati e Porter (2011), uma amostra em que as informações do regressando são disponíveis apenas para algumas observações é conhecida como amostra censurada. Portanto, o modelo Tobit, conhecido também como modelo de regressão censurado, seria o mais indicado porque tem variável dependente limitada, devido à restrição imposta aos valores assumidos pelo regressando. A equação 1, abaixo, foi criada a fim de testar as hipóteses da pesquisa.

$$
I D V_{i}=\beta_{0}+\beta_{1} \text { LnSegi }+\beta_{2} \text { Curti }+\beta_{3} \text { Nani }+\beta_{4} \text { Alav }+\beta_{5} \text { LnAtiv }+ \text { Dise }+\varepsilon
$$

Em que:

IDV= índice de disclosure voluntário;

$\operatorname{LnSeg}_{i}=$ Logaritmo natural da quantidade de seguidores da i-ésima empresa;

Curt $_{i}=$ Quantidade de curtidas por divulgações coletadas da i-ésima empresa durante o ano de 2016;

$\mathrm{Nan}_{\mathrm{i}}=$ Quantidade de analistas da i-ésima empresa;

Alav = Total de dívidas sobre o ativo da empresa;

LnAtiv = Logaritmo natural do ativo total

Dise = Variável dummy que representa as empresas da carteira ISE; e

$\varepsilon_{i}=$ erro da regressão $-\varepsilon_{i} \sim \mathrm{N}\left(0, \sigma_{2}\right)$.

Optou-se por adicionar as variáveis de controle em um primeiro momento por estarem associadas ao disclosure voluntário. Assim, com o intuito de garantir mais robustez ao modelo, foram inseridas as variáveis de controle, a saber: 'endividamento' (Alav) (JENSEN; MECKLING, 1976; MURCIA; SANTOS, 2009), por ser uma variável já testada e validada pela literatura referente aos estudos de disclosure; e 'ativo total' (LnAtiv), para controlar o efeito do tamanho da empresa, assim como fizeram Ahmed e Courtis (1999) e Forte et al. (2014).

Além disso, com o intuito de verificar a diferença no nível de divulgação das empresas que compõem a carteira índice de sustentabilidade empresarial (ISE), foi incluída no modelo uma variável dummy, para representar a presença ou não das empresas nessa carteira, pelo fato de essas empresas já terem certa habitualidade para divulgar informações de caráter voluntário. Assim, a variável "Dise” representa uma variável binária para separar as empresas integrantes do índice de sustentabilidade empresarial. Para mensurar a variável latente (Dise), foi atribuída a numeração "1" para as empresas que faziam parte do ISE, e "0", para os demais casos.

Os efeitos esperados das variáveis a serem analisadas por meio do modelo estatístico estão contidos na Tabela 3 .

Tabela 3 - Efeito esperado das variáveis analisadas

\begin{tabular}{cc}
\hline Variáveis explicativas & Efeito no IDV \\
\hline Curtidas & + \\
Seguidores & + \\
Analista & - \\
\hline
\end{tabular}

Fonte: Elaboração própria (2017)

No tópico seguinte, serão analisados os resultados dos testes estatísticos para verificar a relação entre as principais práticas do disclosure voluntário das empresas, por meio do Twitter e do Facebook, de forma separada, e a demanda dos investidores pela informação via internet. 
Também foi realizado um teste de médias para verificar qual a rede social (Twitter ou Facebook) mais usada para as empresas fazerem disclosure voluntário.

\section{APRESENTAÇÃO E DISCUSSÃO DOS RESULTADOS}

\subsection{Estatísticas descritivas}

Ao investigar o disclosure voluntário das empresas da amostra, observou-se que muitas companhias efetuam disclosure voluntário em suas contas do Twitter e do Facebook, no entanto, aproximadamente, $27 \%$ e $28 \%$, respectivamente, das empresas da amostra não divulgam nenhum tipo de informação considerada voluntária de acordo com o IDV.

A Tabela 4 apresenta a média de disclosure, por categoria, que compõe o índice, o maior valor encontrado para o índice e a referida empresa que o apresentou, além da média geral do IDV do Twitter e do Facebook, o que mostra uma baixa divulgação das empresas, considerando, para o cálculo da média, as que não fazem nenhum tipo de disclosure voluntário. De forma segregada, nos Painéis A e B, são feitas as análises do IDV via Twitter, e nos C e D, é analisado o IDV via Facebook.

Tabela 4 - Composição do IDV

\begin{tabular}{lccccl}
\hline \multicolumn{1}{l}{ PAINEL A - Resultado Geral do IDV no Twitter } & & \\
\hline Categorias & Média & Mínimo & Máximo & Mediana & $\begin{array}{l}\text { Empresa } \\
\text { com maior índice }\end{array}$ \\
\hline Ambiental & $10, \%$ & 0 & $60 \%$ & 0 & Sabesp e Braskem \\
Social & $17 \%$ & 0 & $58 \%$ & $8 \%$ & Sabesp e BRF S.A \\
Econômica & $15 \%$ & 0 & $82 \%$ & $9 \%$ & Gerdau \\
\hline IDV & $14 \%$ & 0 & $54 \%$ & $9 \%$ & Gerdau \\
\hline
\end{tabular}

PAINEL B - Resultado do índice com exclusão das empresas que não efetuaram total disclosure e por categoria

\begin{tabular}{lccccl}
\hline Categorias & Média & Mínimo & Máximo & Mediana & $\begin{array}{l}\text { Empresa } \\
\text { com maior índice }\end{array}$ \\
\hline Ambiental & $14 \%$ & 0 & $60 \%$ & $10 \%$ & Sabesp e Braskem \\
Social & $22 \%$ & 0 & $58 \%$ & $21 \%$ & Sabesp e BRF S.A \\
Econômica & $21 \%$ & 0 & $82 \%$ & $14 \%$ & Gerdau \\
\hline IDV & $19 \%$ & $3 \%$ & $54 \%$ & $14 \%$ & Gerdau \\
\hline PAINEL C - Resultado geral do IDV no Facebook & & & \\
\hline \multicolumn{7}{l}{ Categorias } & Média & Mínimo & Máximo & Mediana & Empresa \\
& $11 \%$ & 0 & $70 \%$ & 0 & Braskem maior índice \\
\hline Ambiental & $14 \%$ & 0 & $50 \%$ & 8 & Usiminas \\
Social & $10 \%$ & 0 & $45 \%$ & 0 & Fibria e Braskem \\
Econômica & $12 \%$ & 0 & $49 \%$ & 9 & Braskem \\
\hline IDV & & & & & \\
\hline
\end{tabular}

PAINEL D - Resultado do índice, por categoria, com exclusão das empresas que não efetuaram disclosure

\begin{tabular}{lccccl}
\hline Categorias & Média & Mínimo & Máximo & Mediana & $\begin{array}{l}\text { Empresa } \\
\text { com maior índice }\end{array}$ \\
\hline Ambiental & $14 \%$ & 0 & $70 \%$ & $10 \%$ & Braskem \\
Social & $20 \%$ & 0 & $50 \%$ & $17 \%$ & Usiminas \\
Econômica & $13 \%$ & 0 & $45 \%$ & $9 \%$ & Fibria e Braskem \\
\hline IDV & $16 \%$ & $3 \%$ & $49 \%$ & $12 \%$ & Braskem \\
\hline
\end{tabular}

Notas: A análise amostral do Twitter contemplou 52 empresas, e a do Facebook ficou em 60 empresas durante o período de janeiro a dezembro de 2016. Cada categoria foi ponderada de acordo com o total de perguntas constante no índice: ambiental 
-10 perguntas; social - 12 perguntas; e econômica -11 perguntas. Assim, a cada pergunta do questionário respondida pelas empresas foi atribuída pontuação "1", e a cada pergunta sem resposta, " 0 ". O painel A apresenta o resultado das estatísticas descritivas utilizando-se as respostas de todas as empresas que tinham Twitter, inclusive as que apresentaram zero no valor do IDV. O painel B apresenta o resultado das estatísticas descritivas com exclusão de empresas que apresentaram o IDV zerado. $\mathrm{O}$ painel $\mathrm{C}$ apresenta o resultado das estatísticas descritivas utilizando-se as respostas de todas as empresas que tinham Facebook, incluindo as que apresentaram zero no valor do IDV. O painel D apresenta o resultado das estatísticas descritivas com exclusão de empresas que apresentaram o IDV zerado.

Ao evidenciar as estatísticas descritivas desconsiderando as empresas com IDV zerado, notam-se resultados afins para a média e a mediana entre as categorias e no resultado geral do índice. A empresa com maior diclosure voluntário que usou o Twitter foi a Gerdau que, expressivamente, divulga muitas informações de caráter econômico. Já a empresa com maior disclosure voluntário via Facebook foi a Braskem, que faz muitas divulgações ambientais e econômicas.

É importante ressaltar que a Braskem faz parte da Carteira do ISE e está entre as empresas que fazem mais divulgações voluntárias tanto por meio do Twitter quanto Facebook, porque, conforme Gray e Bebbington (2000), espera-se um nível maior de divulgação para as organizações que se comprometem com a sustentabilidade. A Sabesp, BRF S.A e a Usiminas, apesar de não comporem a carteira ISE, mantêm uma sólida política socioambiental com projetos focados no apoio à cultura, o que justifica suas pontuações na composição do índice.

As Tabelas 5 e 6 apresentam a estatística descritiva referente às variáveis utilizadas no modelo para o Twitter e para o Facebook, respectivamente.

Tabela 5 - Estatística descritiva das variáveis explicativas (Twitter)

\begin{tabular}{lccccc}
\hline \multicolumn{5}{c}{ Variáveis do modelo } \\
\hline \multicolumn{1}{c}{ Variáveis } & Média & Mediana & Desvio-padrão & Mínimo & Máximo \\
LnSeg & 9,339155 & 9,560004 & 2,436505 & 4,077537 & 13,36138 \\
Curt & 227,0192 & 28,5 & 705,5818 & 0 & 4.855 \\
Nan & 10,88462 & 11 & 3,909034 & 0 & 18 \\
Alav & 0,3313688 & 0,3138528 & 0,1689556 & 0,0309073 & 0,7420586 \\
LnAtiv & 23,83744 & 23,68454 & 1,553687 & 21,45308 & 27.96848 \\
Dise & 0,4038462 & 0 & 0,4954545 & 0 & 1 \\
\hline
\end{tabular}

Fonte: Dados da pesquisa (2017). Notas: Amostra composta de 52 empresas, em que: LnSeg = logaritmo natural de seguidores; curt $=$ curtidas dos seguidores nos twitters das empresas; Nan = número de analistas que acompanham a empresa; Alav = endividamento da empresa; LnAtiv = logaritmo natural de ativo total; e dise: dummy para participação da empresa no ISE e zero nos demais casos.

Observa-se que a média de curtidas (Curt), via Twitter, de informações consideradas como disclosure voluntário pelas empresas da amostra é discrepante em relação à mediana. A distribuição da variável 'curtidas' é muito heterogênea, pois apresenta valores mínimos iguais a zero, já que, para as empresas que apresentarem um IDV zerado, o número de curtidas também é zero. Por outro lado, o número máximo de curtidas (4.855) indica uma razoável visibilidade dada pelos usuários do Twitter ao disclosure voluntário feito pelas empresas.

Quanto ao quantitativo de seguidores, usou-se o logaritmo natural para minimizar a heterogeneidade dos dados. Assim, para a variável LnSeg (Tabela 5), obtiveram-se uma média e uma mediana bem similares. Os valores máximo e mínimo do $\mathrm{LnSeg}$, em relação às variáveis 'Curt' e 'Nan', são mais próximos, e isso ressalta o poder de disseminação de informações, já que, quanto mais seguidores uma empresa tem, mais expressivo será o acompanhamento das divulgações que faz. Existem empresas cujas divulgações são acompanhadas por um total de 18 analistas, enquanto outras, por nenhum.

Tabela 6 - Estatística descritiva das variáveis explicativas (Facebook) 


\begin{tabular}{|c|c|c|c|c|c|}
\hline \multicolumn{6}{|c|}{ Variáveis do modelo } \\
\hline Variáveis & Média & Mediana & Desvio-padrão & Mínimo & Máximo \\
\hline LnSeg & 208,2664 & 10,48768 & 1539,184 & 0,6931472 & 11.932 \\
\hline Curt & 9367,2 & 1391,5 & 19657,95 & 0 & 96.700 \\
\hline Nan & 10,66667 & 11 & 3,899877 & 0 & 18 \\
\hline Alav & 0,3312193 & 0,3082233 & 0,1805407 & 0,0309073 & 0,7683502 \\
\hline LnAtiv & 23,61063 & 23,38875 & 1,551516 & 21,42565 & 27,96848 \\
\hline Dise & 0,35 & 0 & 0,4809947 & 0 & 1 \\
\hline
\end{tabular}

Fonte: Dados da pesquisa (2017). Notas: Amostra composta de 60 empresas, em que: $L n S e g=$ logaritmo natural de Seguidores; Curt $=$ curtidas dos seguidores nos Facebooks das empresas; Nan = número de analistas que acompanham a empresa no mercado; Alav = endividamento da empresa; LnAtiv = logaritmo natural de Ativo Total; e Dise: dummy para participação da empresa no ISE e zero nos demais casos.

De forma similar ao que foi encontrado na estatística descritiva das variáveis coletadas no Twitter, a variável Curt (Tabela 6) apresenta uma distribuição muito heterogênea, uma vez que o desvio-padrão é maior, cerca de 13 vezes, em relação ao valor da média, a qual é bem discrepante em relação à mediana. Em contrapartida, os valores máximos, tanto da variável Curt quanto da variável $\mathrm{LnSeg}$, demonstram o poder de alcance das informações divulgadas pelas empresas via Facebook. Por fim, grande parte das empresas que tiveram seus disclosure via Twitter investigados também tiveram seus disclosure por meio do Facebook analisado. Por isso o quantitativo máximo de analistas que seguem a empresa é de 18, e o mínimo, de zero. Isso indica uma concentração de analistas que acompanham determinada empresa em detrimento de outras.

\subsection{Análise do disclosure voluntário via Twitter e Facebook}

\subsubsection{Análise do disclosure voluntário via Twitter}

Incialmente foi avaliado a direção e a intensidade da correlação entre as variáveis que compõem o modelo de regressão. Para tanto, foi rodada a correlação de Spearman, como demonstrado na Tabela 7 abaixo.

Tabela 7 - Matriz de correlação de Spearman entre as variáveis estudadas (Twitter)

\begin{tabular}{|c|c|c|c|c|c|c|c|c|c|c|c|}
\hline & $I D V$ & & LnSeg & & Curt & & Nan & & Alav & LnAtiv & Dise \\
\hline$I D V$ & 1 & & & & & & & & & & \\
\hline LnSeg & 0,0596 & & 1 & & & & & & & & \\
\hline Curt & 0,7331 & $* * *$ & 0,3642 & $* * *$ & 1 & & & & & & \\
\hline Nan & 0,0293 & & 0,1963 & & 0,2014 & & 1 & & & & \\
\hline Alav & 0,0046 & & 0,0875 & & $-0,0278$ & & 0,2470 & $*$ & 1 & & \\
\hline LnAtiv & 0,4592 & $* * *$ & 0,2952 & $* *$ & 0,5725 & $* * *$ & 0,3128 & $* *$ & 0,1844 & 1 & \\
\hline Dise & 0,2468 & $*$ & 0,2363 & $*$ & 0,3283 & $* *$ & 0,0695 & & $-0,1058$ & 0,1763 & 1 \\
\hline
\end{tabular}

Notas: $*, * * * * *$ representam a significância estatística a $10 \%, 5 \%$ e $1 \%$.

De modo geral, todas as variáveis independentes não apresentam correlações positivas acima de 50\%, exceto pela variável Curt. Isso pode ser um indicativo de que o modelo não terá problemas com muticolineariedade entre as variáveis explicativas. Quanto à correlação das variáveis independentes com a dependente (IDV), nota-se os coeficientes positivos e significantes das variáveis 'Curt' e 'LnSeg', com destaque para a variável Curt, que tem uma correlação representativa com o IDV. Como a variável 'Dise' é significante e positiva, isso indica que as empresas enquadradas no índice de sustentabilidade empresarial da B3 apresentam correlação positiva com o índice de disclousure voluntário. 
A seguir, na Tabela 8, são apresentados os resultados da regressão Tobit, utilizando-se o índice de disclosure voluntário como variável dependente e a quantidade de curtidas, de seguidores e de analistas de mercado, a alavancagem e o ativo total como variáveis independentes. Nesse modelo, utilizou-se uma variável dummy, com o objetivo de identificar o comportamento das empresas que compõem a carteira índice de sustentabilidade empresarial (ISE) em relação às demais, conforme a equação 1.

É possível perceber, pela Tabela 8, Painel A, que as variáveis representativas de curtidas - 'Curt'; endividamento - 'Alav'; e tamanho da empresa - 'LnAtiv', mantêm relacionamento positivo com a variável dependente, e as variáveis representativas de seguidores - 'LnSeg'; e a quantidade de analistas que acompanham as empresas no mercado - 'Nan' - mantêm relacionamento inverso.

Tabela 8 - Resultado da regressão Tobit (Twitter)

\begin{tabular}{lccccc}
\hline \multicolumn{7}{c}{ PAINEL A } \\
\hline Variáveis & Coeficiente & Erro-padrão & Estatística t & Intervalo de confiança \\
LnSeg & $-0,0000^{* * *}$ & 0,0000 & $-2,7500$ & 0,0000 & 0,0000 \\
Curt & $0,0001^{* *}$ & 0,0000 & 2,5600 & 0,0000 & 0,0002 \\
Nan & $-0,0039$ & 0,0066 & $-0,5800$ & $-0,0172$ & 0,0095 \\
Alav & 0,0942 & 0,1649 & 0,5700 & $-0,2376$ & 0,4261 \\
LnAtiv & $0,0341^{*}$ & 0,0189 & 1,8000 & $-0,0040$ & 0,0722 \\
Dise & $0,1044^{*}$ & 0,0542 & 1,9300 & $-0,0047$ & 0,2135 \\
Constante & $-0,7130$ & 0,4306 & $-1,6600$ & $-1,5799$ & 0,1538 \\
\hline \multicolumn{7}{c}{ PAINEL B } \\
\hline Pseudo R & 1,5762 & \multicolumn{7}{c}{ LR chi2 } \\
$\mathrm{N}^{\mathrm{o}}$ de Obs & 52 & Prob>Chi2 & 18,29 \\
\hline
\end{tabular}

Notas:*,**, *** representam a significância estatística a $10 \%, 5 \%$ e $1 \%$, respectivamente. Amostra composta de 52 observações, em que: $\mathrm{LnSeg}=$ logaritmo natural de seguidores (Quantitativo de usuário do Twitter que seguem a página da empresa); Curt = curtidas dos seguidores nos Twitters das empresas; Nan = número de analistas que acompanham a empresa no mercado; Alav = endividamento da empresa; LnAtiv = logaritmo natural do ativo total da empresa; Dise = variável que evidencia se a empresa faz parte do índice de responsabilidade empresarial.

Na Tabela 8, os coeficientes das variáveis $L n S e g$ e Curt são significantes e explicam o comportamento do IDV, embora o sinal negativo do LnSeg seja contrário ao esperado, indicando que as empresas com menor número de seguidores são as que mais divulgam informações de caráter voluntário. No que concerne à visualização das informações voluntárias (Curt), há uma relação direta entre a quantidade curtidas dos seguidores/usuário do Twitter e o número de informações voluntárias divulgadas. A variável LnAtiv foi significante a $10 \%$ e positivamente relacionada ao IDV. Isso sugere que as grandes empresas, notadamente as com os maiores valores em ativos totais, são as que mais se utilizam do Twitter como ferramenta para propagar informações voluntárias sobre aspectos ambientais, sociais e econômicos. Esse fato é congruente com os trabalhos de Ahmed e Courtis (1999) e Forte et al. (2014), que constataram que o tamanho da empresa é considerado uma variável relevante na explicação do disclosure voluntário.

Como esperado, a participação das empresas no ISE foi significante e positivamente relacionada ao IDV. Isso sugere que a habitualidade das empresas da amostra de divulgarem informações voluntárias por meio dos canais comuns de comunicação também está presente quando elas utilizam redes sociais, como o Twitter. Quanto às demais variáveis explicativas, nenhuma se mostrou significante, porém, com exceção da variável 'seguidores', as variáveis 'curtidas' e 'tamanho' apresentaram a relação esperada pela literatura. Quanto à variável 'Nan', não foi significante e, dessa forma, não é possível afirmar que a direta e ampla disseminação de informações via Twitter seria mais benéfica para as empresas que são pouco visadas, do que 
para as empresas que são mais acompanhadas pelos meios tradicionais de disseminação de informações, como os analistas de mercado (BLANKESPOOR et al., 2013).

\subsubsection{Análise do disclosure voluntário via Facebook}

Seguidamente à análise do disclosure via Twitter, neste tópico, foi analisado o disclosure voluntário realizado por 60 empresas por meio do Facebook. Na Tabela 9, apresentase a matriz de correlação de Spearman, visando verificar a direção (negativa ou positiva) e a intensidade da correlação entre as variáveis do modelo.

Tabela 9 - Matriz de correlação de Spearman entre as variáveis estudadas (Facebook)

\begin{tabular}{|c|c|c|c|c|c|c|c|c|c|c|c|c|c|}
\hline & $I D V$ & & LnSeg & & Curt & & Nan & & Alav & & LnAtiv & & Dise \\
\hline$I D V$ & 1 & & & & & & & & & & & & \\
\hline LnSeg & 0,2573 & $* *$ & 1 & & & & & & & & & & \\
\hline Curt & 0,6574 & $* * *$ & 0,4551 & $* * *$ & 1 & & & & & & & & \\
\hline Nan & 0,2435 & $*$ & 0,0835 & & 0,2258 & * & 1 & & & & & & \\
\hline Alav & 0,3827 & $* * *$ & 0,1652 & & 0,2595 & $* *$ & 0,2469 & $*$ & 1 & & & & \\
\hline LnAtiv & 0,5886 & $* * *$ & 0,3435 & $* * *$ & 0,5544 & $* * *$ & 0,4028 & $* * *$ & 0,1690 & & 1 & & \\
\hline Dise & 0,3706 & $* * *$ & 0,4227 & $* * *$ & 0,4023 & $* * *$ & 0,2928 & $* *$ & 0,3945 & $* * *$ & 0,3884 & $* * *$ & 1 \\
\hline
\end{tabular}

Notas: *,**,*** representam a significância estatística a $10 \%, 5 \%$ e $1 \%$, respectivamente.

Perceptivelmente, o mesmo comportamento verificado na Tabela 7 também pode ser observado na Tabela 9, em que as variáveis explicativas não apresentam altas correlações entre si. O maior coeficiente de correlação é, notadamente, o da variável Curt, seguido do coeficiente da variável LnAtiv, ainda que essas mesmas duas variáveis apresentem uma correlação de 0,55 entre si, o que sugere que grandes empresas são também as mais visualizadas (curtidas) em termo de informações voluntárias divulgadas.

$\mathrm{Na}$ Tabela 10, apresentam-se os resultados da regressão Tobit para a amostra de empresas cuja página do Facebook foi analisada para construir o IDV. Nessa amostra, o quantitativo de empresas que têm Facebook ativo, mas não divulgam nenhum tipo de diclosure voluntário considerado no IDV, foi de cerca de $28 \%$ da amostra.

Tabela 10 - Resultado da regressão Tobit (Facebook)

\begin{tabular}{lccccc}
\hline \multicolumn{5}{c}{ PAINEL A } \\
\hline Variáveis & Coeficiente & Erro-padrão & Estatística t & Intervalo de confiança \\
LnSeg & $-0,0000^{*}$ & 0,0000 & $-1,7100$ & 0,0000 & 0,0000 \\
Curt & $0,0000^{* *}$ & 0,0000 & 2,0300 & 0,0000 & 0,0000 \\
Nan & 0,0013 & 0,0048 & 0,2700 & $-0,0083$ & 0,0109 \\
Alav & $0,2671^{* *}$ & 0,1051 & 2,5400 & 0,0564 & 0,4778 \\
LnAtiv & $0,3258^{* *}$ & 0,0133 & 2,4500 & 0,0060 & 0,0592 \\
Dise & $0,3243^{* *}$ & 0,4146 & 0,7800 & $-0,0507$ & 0,1155 \\
Constante & $-0,8157^{* *}$ & 0,3086 & $-2,6400$ & $-1,4343$ & $-1,1970$ \\
\hline Pseudo $\mathrm{R}^{2}$ & $-4,2596$ & \multicolumn{5}{c}{26,74} \\
$\mathrm{~N}^{\mathrm{o}}$ de Obs & 60 & LR chi2 & $-4,2596$ \\
\hline
\end{tabular}

Notas:*, **, *** representam a significância estatística a $10 \%, 5 \%$ e 1\%, respectivamente. Amostra composta de 60 observações, em que: $\mathrm{LnSeg}=$ logaritmo natural de seguidores (pessoas que seguem os posts das empresas); Curt = curtidas nas informações consideradas voluntárias ; Nan = número de analistas que acompanham a empresa no mercado; Alav = endividamento da empresa; LnAtiv = logaritmo natural de ativo total das empresas; Dise = variável que evidencia se a empresa faz parte do índice de responsabilidade empresarial. 
Analisando as variáveis de interesse, nota-se que o LnSeg e a quantidade de curtidas foram significantes.A relação positiva entre o IDV e a quantidade de curtidas (Curt) indicaria que o ato de curtir uma divulgação voluntária pelo usuário do Facebook seria um indicativo de que a empresa tem usuários que estão realmente atentos ao tipo de divulgação que ela está fazendo. No tocante ao $\mathrm{LnSeg}$, apresentou uma relação negativa inesperada, o que sugere que, diferentemente do seguidor que curte a informação, quanto maior o quantitativo de seguidores menor o nível de disclosure voluntário das empresas via Facebook. Isso, em parte, pode ser explicado pelo fato de empresas como a CVC Brasil, as Lojas Renner, as Lojas Americanas, o Bradesco e outras terem uma expressiva quantidade de seguidores, no entanto são empresas que só usam o Facebook para fazer propaganda dos seus produtos e serviços.

As variáveis 'alavancagem' e 'ativo total' são significantes e positivamente relacionadas ao IDV. Assim, da mesma forma que o tamanho da empresa pode explicar o disclosure voluntário via Twitter, isso também foi encontrado no caso do Facebook. Já a alavancagem indica que as empresas mais endividadas realizam maior disclosure voluntário tanto pelos canais tradicionais (MURCIA; SANTOS, 2009), como por meio do Facebook, possivelmente pelo fato de já ser política da empresa executar maior disclosure por meio de outros canais. Isso tenderia a refletir no uso de redes sociais como um canal adicional a essa prática corporativa.

\subsection{Teste de médias}

Neste tópico, investigou-se a existência de diferença entre o nível de disclosure voluntário feito, pelas empresas, nas duas redes sociais investigadas. Para tanto, foram executados os procedimentos para testar a normalidade e a heterogeneidade das variáveis IDVt (índice de disclosure voluntário via Twitter) e IDV-f (índice de disclosure voluntário via Facebook). Como se constatou que o pressuposto da normalidade não foi cumprido, foi usado o teste de Wilcoxon para amostras independentes, embora o resultado do teste $t$ também seja evidenciado para efeito de comparação.

Tabela 11 - Teste de médias emparelhadas

\begin{tabular}{ccccccccc}
\hline & Obs & Média & Desvio-padrão & \multicolumn{2}{c}{ Intervalo de confiança } & Tipo & P-valor & Teste \\
\hline IDV-t & 52 & 0,1424 & 0,1594 & 0,09798 & 0,18676 & Teste $\mathrm{t}$ & 0,5977 & $\mathrm{t}=1,091$ \\
IDV-f & 60 & 0,1133 & 0,1215 & 0,08196 & 0,14474 & Wilcoxon & 0,8665 & $\mathrm{z}=0,496$ \\
\hline
\end{tabular}

Fonte: Dados da pesquisa (2017).

O resultado do $t$ teste e do teste de Wilcoxon indica que não há diferenças significativas entre o disclosure voluntário realizado pelas empresas tanto por meio do Facebook quanto do Twitter. Esse resultado pode ser justificado pela perspectiva de que empresas que participam ativamente de uma rede social também acabem usando outras redes no mesmo sentido. Isso poderia indicar uma tendência de adesão e contínuo uso de redes sociais como ferramenta de divulgação de informações consideradas importantes pelas empresas, já que esse canal de disclosure é usado de forma voluntária.

\section{CONSIDERAÇÕES FINAIS}

No Brasil, o disclosure de informações via redes sociais apresenta-se em caráter voluntário, e muitas empresas divulgam suas ações e projetos discricionariamente por meio de tais canais. Assim, fundamentado na Teoria do Disclosure Voluntário, o estudo teve o intuito de investigar a divulgação de informações sociais, ambientais e econômicas feitas em 2016 por meio do Twitter e do Facebook das empresas que faziam parte da carteira do IBrX100 divulgada no início de 2017. 
Representada pela quantidade de seguidores, a demanda dos usuários por informações corporativas via redes sociais não apresentou a relação esperada no tocante ao disclosure de informações voluntárias pelas empresas. Ao que parece, uma maior quantidade de seguidores estaria concentrada em empresas que divulgam menores índices de informações ambientais, sociais e econômicas. Argumenta-se que, mesmo sem um teste explícito, essas empresas são as que mais divulgam/publicam outros tipos de informação (produtos, promoções) em suas redes sociais. No tocante ao número de curtidas efetuadas nas informações consideradas voluntárias, a relação positiva com o IDV sugere que o comportamento de visualização por parte do usuário do Facebook e do Twitter de informações ambientais, sociais e econômicas seria um fator determinante do nível de disclosure de informações voluntárias realizado pelas empresas. Em outras palavras, a quantidade de curtidas possibilitaria à empresa visualizar a ampliação do alcance informacional de suas divulgações, já que o número de curtidas é um feedback do tipo de informação à qual os usuários estão reagindo.

Por fim, a segunda hipótese era de verificar se as empresas que são menos visadas pelos analistas de mercado se aproveitariam do custo baixo e da tempestividade da informação divulgada em redes sociais para realizar seus disclosures. Encontrou-se a relação esperada, ou seja, quanto mais analistas a empresa tem, menos ela realiza disclosure voluntário no Facebook e no Twitter. Todavia essa relação não se mostrou estatisticamente significante. É válido ressaltar que há um considerável aumento do uso de redes sociais pelas empresas, e o tipo de informação que elas publicam é um ato discricionário da empresa que merece atenção devido ao seu poder de afetar a percepção dos usuários dessas mídias sociais quanto aos tipos de disclosure (Ex: desempenho financeiro) que são altamente regulados.

Quanto às limitações, este estudo só analisou as empresas presentes na carteira teórica do IBrX100 e que tinham conta de Twitter e/ou de Facebook ativas, sem analisar outras mídias sociais. Então, os resultados devem ser analisados com cautela, devido ao curto período e à limitação amostral. Essas limitações podem ser exploradas por outras pesquisas futuras.

\section{REFERÊNCIAS}

ABDELSALAM, O. H.; BRYANT, S. M.; STREET, D. L. An examination of the Comprehensiveness of Corporate London-Listed Companies. Journal of International Accounting Research, v. 6, n. 2, p. 1-33, 2007.

AHMED, K.; COURTIS, J. K. Associations between corporate characteristics and disclosure levels in annual reports: a meta-analysis. British Accounting Review, v. 31, p. 35-61, 1999.

AKERLOF, G. A. The market for "Lemons": quality uncertainty and the market mechanism. The Quarterly Journal of Economics, v. 84, n. 3, p. 488-500, 1970.

ALBANEZ, T.; VALLE, M. R. DO. Impactos da assimetria de informação na estrutura de capital de empresas brasileiras abertas. Revista Contabilidade \& Fiananças, v. 20, n. 51, p. 6-27, 2009.

ALENCAR, R. C. DE. Nível de disclosure e custo de capital próprio no mercado brasileiro. 2007. Tese de Doutorado, Universidade de São Paulo, São Paulo, SP, Brasil, 2007.

ALEXA. The top 500 sites on the web. . [S.1: s.n.]. , 2013

ALTOÉ, S. M. L.; PANHOCA, L.; ESPEJO, M. M. DOS S. B. Índice de disclosure ambiental (IDA): análise da aplicação de indicador desenvolvido a partir da ótica de especialistas no Brasil. Anais do XV Congresso USP Controladoria e Contabilidade. São Paulo, SP, 
Brasil: [s.n.]. , 2015

ARRUDA, M. P. et al. Divulgação de informações por meio da internet: serão as redes sociais capazes de reduzir a assimetria informacional entre empresas e investidores? Revista Evidenciação Contábil \& Finanças, v. 3, n. 2, p. 27-41, 2015.

ARRUDA, M. P.; GIRÃO, L. F. DE A. P.; LUCENA, W. G. L. Assimetria informacional e o preço das ações: análise da utilização das redes sociais nos mercados de capitais brasileiro e norte-americano. R. Cont. Fin. - USP, v. 26, n. 69, p. 317-330, 2015.

BERETTA, S.; BOZZOLAN, S. Quality versus quantity : the case of forward-looking disclosure. Journal of Accounting Auditing and Finance. [S.1.]: Social Science Research Network. , 2007

BERTOMEU, J.; MAGEE, R. P. Mandatory disclosure and asymmetry in financial reporting. Journal of Accounting and Economics, v. 59, n. 2-3, p. 284-299, 2015.

BLANKESPOOR, E.; MILLER, G. S.; WHITE, H. D. The role of dissemination in market liquidity: evidence from firms's use of twitter. The Accounting Review, v. 89, n. 1, p. 79$112,2013$.

BOTOSAN, C. A. Disclosure level and the cost of equity capital. The Accounting Review, v. 72, n. 3, p. 323-349, 1997.

BOTOSAN, C. A.; PLUMLEE, M. A. Assessing alternative proxies for the Expected Risk Premium. The Accounting Review, v. 80, n. 1, p. 21-53, 2005.

BUSHEE, B. J.; NOE, C. F. Corporate Disclosure Practices, institutional investors , and Stock Return Volatility. Journal of Accounting Research, v. 38, n. 2000, p. 171-202, 2000.

BUSHMAN, R. et al. Financial accounting information, organizational complexity and corporate governance systems. Journal of Accounting and Econ, v. 37, p. 167-201, 2004.

CALLADO, A. L. C. Modelo de mensuração de sustentabilidade empresarial: uma aplicação em vinícolas localizadas na Serra Gaúcha. 2010. Tese de Doutorado, Universidade Federal do Rio Grande do Sul, Porto Alegre, RS, Brasil, 2010.

CHEN, K. C. W.; CHEN, Z.; WEI, K. C. J. Disclosure, corporate governance and the cost of equity capital: evidence from Asia's emerging markets. [S.l: s.n.], 2003.

DRAKE, M. S.; THORNOCK, J. R.; ROULSTONE, D. T. Investor information demand: evidence from google searches around earnings announcements. [S.l: s.n.], 2012.

DYE, R. A. An evaluation of "“ essays on disclosure "” and the disclosure literature in accounting. Journal of Accounting and Economics, v. 32, p. 181-235, 2001.

DYE, R. A. Disclosure of Nonproprietary Information. Journal of Accounting Research, v. 23, n. 1, p. 123-146, 1985.

FORTE, L. M. et al. Determinantes do disclosure voluntário: um estudo no setor bancário brasileiro. Anais do XXXVIII EnANPAD. Rio de Janeiro, RJ, Brasil: [s.n.]. , 2014. 
GIRÃO, L. F. DE A.; MARTINS, O. S.; PAULO, E. Avaliação de empresas e probabilidade de negociação com informação privilegiada no mercado brasileiro de capitais. Revista de Administração, v. 49, n. 3, p. 462-475, 2013.

GRAY, R.; BEBBINGTON, J. Environmental accounting managerialism and sustainability: is the planet safe in the hands of business and accounting? Advances in Enrironmental Accounting \& Management, v. 1, p. 1-44, 2000.

GUJARATI, D. N.; PORTER, D. C. Econometría. Quinta edi ed. [S.l: s.n.], 2011.

HAIL, L. The impact of voluntary corporate disclosures on the ex ante cost of capital for swiss firms. The European Accounting Review, v. 11, n. 4, p. 741-773, 2002.

HEALY, P. M.; HUTTON, A. M. Y. P.; PALEPU, K. G. Stock performance and intermediation changes surrounding sustained increases in disclosure. Contemporary Accounting Research, v. 16, n. 3, p. 485-520, 1999.

HEALY, P. M.; PALEPU, K. G. Information asymmetry, corporate disclosure, and the capital markets: a review of the empirical disclosure literature. Journal of Accounting and Economics, v. 31, n. 1-3, p. 405-440, 2001.

HUGHES, J. S.; PAE, S. Voluntary disclosure of precision information. Journal of Accounting and Economics, v. 37, p. 261-289, 2004.

JENSEN, M. C.; MECKLING, W. H. Theory of the Firm: managerial behavior, agency costs and ownership structure. Journal of Financial Economics, v. 3, n. 4, p. 160-305, 1976. Disponível em: <http://dx.doi.org/10.1016/0304-405X(76)90026-X〉.

LEUZ, C.; VERRECCHIA, R. E. The economic consequences increased disclosure. Journal of Accounting Research, v. 38, n. 2000, p. 91-124, 2000.

MACAGNAN, C. B. Evidenciação voluntária: fatores explicativos da extensão da informação sobre recursos intangíveis. (Portuguese). n. 50, p. 46-61, 2009. Disponível em: $<$ http://search.ebscohost.com/login.aspx?direct=true\&db=a9h\&AN=44451553\&lang=ptbr\&site=ehost-live $>$.

MENDES-DA-SILVA, W. et al. Disclosure via website corporativo: um exame de informações financeiras e de governança no mercado brasileiro. RAE, v. 49, n. 2, p. 190-205, 2009.

MURCIA, F. D.-R.; SANTOS, A. DOS. Fatores determinantes do nível de disclosure voluntário. Revista de Educação e Pesquisa em Contabilidade, v. 3, n. 2, p. 72-95, 2009.

PAE, S. Selective disclosures in the presence of uncertainty about information endowment. Journal of Accounting and Economics, v. 39, p. 383-409, 2005.

PAULO, E. Manipulação das informações contábeis: uma análise teórica e empírica sobre os modelos operacionais de detecção de gerenciamento de resultados. 2007. 260 f. Faculdade de Economia, Administração e Contabilidade - Universidade de São Paulo, São Paulo, 2007.

RUBIN, A.; RUBIN, E. Informed investors and the Internet. Journal of Business Finance \& 
Accounting, v. 37, p. 841-865, 2010.

SILVA, M. D. DE O. P.; LUCENA, W. G. L. Informações socioambientais em rede social e o reflexo na precificação das ações integrantes do IBrX100. Anais do XV Congresso USP de Controladoria e Contabilidade, jul. 2015.

SILVA, W. M. DA; MARTELANC, R. Relacionamento com investidores com uso do website corporativo: análise empírica das empresas brasileiras listadas na Bovespa. Reflexão Contábil, v. 25, n. 3, p. 41-49, 2006.

VERRECCHIA, R. E. Discretionary Disclosure. Journal of Accounting and Economics, v. 5, p. 179-194, 1983.

VERRECCHIA, R. E. Essays on disclosure. Journal of Accounting and Economics, v. 32, n. 1-3, p. 97-180, 2001.

WELKER, M. Disclosure policy, information asymmetry, and liquidity in equity markets *. v. I, n. 2, 1995.

ZHANG, J. Voluntary information disclosure on social media. Decision Support Systems, v. 73, p. 28-36, 2015.

ZHOU, H. The impact of increased accounting disclosure on information asymmetry: a case of implementing new auditing standards in emerging markets. . [S.1.]: Social Science

Research Network, 2004. 